\title{
Harmine induces anticancer activity in breast cancer cells via targeting TAZ
}

\author{
YU DING ${ }^{1,2^{*}}$, JINRONG HE $^{1,2^{*}}$, JUAN HUANG $^{3^{*}}$, TONG YU $^{4}$, XIAOYAN SHI ${ }^{1,2}$, \\ TIANZHU ZHANG ${ }^{1,2}$, GE YAN $^{1,2}$, SHANSHAN CHEN $^{1,2}$ and CAIXIA PENG ${ }^{1,2}$ \\ ${ }^{1}$ Key Laboratory for Molecular Diagnosis of Hubei Province; ${ }^{2}$ Central Laboratory; \\ ${ }^{3}$ Department of Nephrology, The Central Hospital of Wuhan, Tongji Medical College, \\ Huazhong University of Science and Technology, Wuhan, Hubei 430014; ${ }^{4}$ Department of \\ Traditional Chinese Medicine, Humanwell Healthcare (Group) Co., Ltd., Wuhan, Hubei 430075, P.R. China
}

Received October 26, 2018; Accepted March 27, 2019

DOI: $10.3892 /$ ijo.2019.4777

\begin{abstract}
Harmine (HM) is a $\beta$-carboline alkaloid found in multiple medicinal plants. It has been used in folk medicine for anticancer therapy; however, the molecular mechanism of $\mathrm{HM}$ on human breast cancer remains unclear. Transcriptional co-activator with PDZ-binding motif (TAZ), also known as WW domain-containing transcription regulator 1 , serves an important role in the carcinogenesis and progression of breast cancer. The aim of the present study was to elucidate the potential anticancer activity and mechanism of HM in breast cancer, in vitro and in vivo. Cell proliferation was measured using a CCK-8 assay, apoptotic activity was detected by flow cytometry and DAPI staining, and cell migration was examined using a wound healing assay. The expression of proteins, including extracellular signal-regulate kinase (Erk), phosphorylated (p-) Erk, protein kinase B (Akt), p-Akt, B-cell lymphoma 2 (Bcl-2) and $\mathrm{Bcl}-2$-associated $\mathrm{X}$ protein $(\mathrm{Bax})$, were determined by western blotting. The mRNA expression of TAZ was detected using reverse transcription-quantitative polymerase chain reaction analysis. The expression of proteins in mouse tumor tissues were examined by immunohistochemistry. HM
\end{abstract}

Correspondence to: Dr Shanshan Chen and Dr Caixia Peng, Key Laboratory for Molecular Diagnosis of Hubei Province, The Central Hospital of Wuhan, Tongji Medical College, Huazhong University of Science and Technology, 26 ShengLi Street, Jiangan, Wuhan, Hubei 430014, P.R. China

E-mail: chenshanshan@zxhospital.com

E-mail: echo_by_me@126.com

*Contributed equally

Abbreviations: HM, harmine; FBS, fetal bovine serum; MAPKs, mitogen-activated protein kinases; Bcl-2, B-cell lymphoma 2; Bax, Bcl-2-associated X protein; Akt, protein kinase B; Erk, extracellular signal-regulated protein kinase

Key words: harmine, transcriptional co-activator with PDZ-binding motif, breast cancer, proliferation, migration, apoptosis significantly suppressed cellular proliferation and migration, promoted apoptosis in vitro and inhibited tumor growth in vivo. In addition, HM significantly decreased the expression of TAZ, p-Erk, p-Akt and Bcl-2, but increased that of Bax. The overexpression of TAZ in breast cancer cells inhibited the antitumor effect of HM. In conclusion, HM was found to induce apoptosis and prevent the proliferation and migration of human breast cancer cell lines, possibly via the downregulation of TAZ.

\section{Introduction}

Breast cancer is the most frequent type of cancer among women and is the second leading cause of cancer-associated mortality in the female population of the United States (1). In the last two decades, the prognosis of patients with breast cancer has improved due to lifestyle modification, early detection, prophylactic mastectomy and advanced therapies (2). However, the curative effects of conventional chemotherapeutic agents are not satisfactory, and certain patients with advanced-stage breast cancer exhibit resistance to existing chemotherapeutic drugs (3). The risk factors of breast cancer are multiple and complicated, and the molecular mechanisms underlying its pathogenesis remain to be elucidated. Therefore, there has been increasing interest in identifying alternative chemotherapeutic drugs with novel anticancer mechanisms.

Harmine (HM) is a $\beta$-carboline alkaloid that was originally isolated from seeds of Peganum harmala and Banisteriopsis caapi in 1847 (4). HM is widely distributed in various medicinal plants and has long been used in folk medicine in the Middle East and Asia (5). Studies have demonstrated that HM exhibits significant antitumor activities in vitro and in vivo (6), including inhibiting proliferation (6), migration (7) and invasion (8), promoting apoptosis (9) and preventing tumorigenesis. HM inhibits the growth of several types of cancer, including lung (10), gastric (9), breast (11) and hepatic cancer (12). It arrests the cell cycle at the G0/G1 phase (13) and decreases the cyclin-dependent kinase activity (14). HM induces autophagy and apoptosis through the protein kinase B (Akt)/mammalian target of rapamycin (mTOR) and extracellular signal-regulated kinase (ERK)1/2 signaling pathways, increases the expression 
of pro-apoptotic factors, including P53, caspases 3/8/9, B-cell lymphoma 2 (Bcl-2)-associated X protein (Bax), and $\mathrm{BH} 3$-interacting domain death agonist (Bid), and reduces the level of pro-inflammatory cytokines, including TNF- $\alpha$, IL-6 and granulocyte-macrophage colony-stimulating factor, in melanoma and gastric cancer (7). HM induces autophagy via increasing LC3-II and downregulating P62 in a dose-dependent manner in B16 cells (15). It can also suppress the expression of pro-metastatic genes, including matrix metalloproteinase-9, ERK and vascular endothelial growth factors, to inhibit melanoma cell invasion (16).

Transcriptional co-activator with PDZ-binding motif (TAZ) was initially identified as a 14-3-3 binding protein (17). Due to the lack of a DNA-binding domain, TAZ is not a transcription factor, but it can function as a transcriptional regulator though its transactivation domain. In addition to its interaction with 14-3-3, it has been reported to interact with multiple proteins, including thyroid transcription factor-1 (18), myogenic differentiation 1 (19), Smads (20), core-binding factor $\alpha 1$ /Runt-related transcription factor 2 (21), transcriptional enhancer factor-1 (22), peroxisome proliferator-activated receptor (23) and TEA domains (24). Serving as a transcriptional coactivator, TAZ is important in osteoblastic, myogenic and adipogenic differentiation (25). TAZ was initially identified as an oncogenic protein in non-small cell lung cancer in 2011 (26). Accumulating studies have confirmed that the expression of TAZ is elevated in various types of human cancer, including colorectal cancer (27), glioblastoma (28) and breast cancer $(29,30)$. The overexpression of TAZ leads to cancer cell behavior, including, but not limited to, growth-factor-independent proliferation (31) and resistance to chemotherapeutics (32). It promotes epithelial-mesenchymal transition (30), cell proliferation, migration, invasion, tumorigenesis and tumor formation in xenograft models (33), suggesting an oncogenic role of TAZ in the development of human cancer. Studies have shown that TAZ knockdown in MCF7 and Hs578T cells inhibited cell migration and invasion. The knockdown of TAZ in MCF7 cells also suppressed cell growth in vitro and in vivo (34). These data suggest that TAZ promotes cell proliferation, migration and invasion in breast cancer.

Considering the crucial roles of TAZ in breast cancer, the present study hypothesized that HM induces anticancer activity in breast cancer through the regulation of TAZ. The present study first showed that HM inhibited proliferation and migration, and promoted apoptosis in breast cancer cell lines, confirming its anticancer activity. Subsequently, it was found that the overexpression of TAZ was able to rescue HM-induced cellular toxicity. The results of the present study showed that HM exerted anticancer activity through inhibiting the expression and activity of TAZ.

\section{Materials and methods}

Preparation and chemical analysis of HM. HM (Fig. 1) was separated from the seeds of Peganum nigellastrum Bunge by column chromatography on AB-8 Macroporous Resin eluted with gradient ethyl alcohol and water. Furthermore, HM was isolated by preparative high-performance liquid chromatography (Fig. S1). Structure identification and the purity (>98\%) of this compound (Figs. S2-8) were identified by spectroscopic methods and compared with the reported spectral data.

Cell culture and HM treatment. The MDA-MB-231 and MCF-7 human breast cancer cell lines were obtained from the Shanghai Institute of Biochemistry and Cell Biology (Shanghai, China). The cells were cultured in DMEM (cat. no. C11965500BT; Thermo Fisher Scientific, Inc., Waltham, MA, USA) supplemented with 10\% FBS (cat. no. 10100154; Gibco; Thermo Fisher Scientific, Inc.) and $100 \mu \mathrm{g} / \mathrm{ml}$ streptomycin at $37^{\circ} \mathrm{C}$ in an incubator with $5 \% \mathrm{CO}_{2}$. A 200-mM stock solution of $\mathrm{HM}$ was prepared in DMSO (cat. no. V900090; Merck KGaA, Darmstadt, Germany) and diluted in culture media as necessary, to achieve the final desired experimental concentrations.

Cell transfection. The pCMV6-TAZ expression vector and pCMV6-control vector were purchased from OriGene Technologies, Inc. (Rockville, MD, USA; cat. no. Rc204082). MDA-MB-231 and MCF-7 cells were cultured in 96-well plates at a density of $5 \times 103$ cells per well for $24 \mathrm{~h}$ at $37^{\circ} \mathrm{C}$, and then transfected with pCMV6-TAZ or pCMV6-control using Lipofectamine 3000 (cat. no. L3000-015; Thermo Fisher Scientific, Inc.) for 2 days. The transfected cells were visualized using a fluorescence microscope.

$C C K-8$ assay. The cells were plated in 96-well plates at a density of $5 \times 10^{3}$ cells per well. Following $24 \mathrm{~h}$ of incubation, the cells were left untreated or treated with $\mathrm{HM}$ at 50,100 or $150 \mu \mathrm{M}$ for 24,48 or $72 \mathrm{~h}$, followed by the addition of $10 \mu \mathrm{l}$ CCK- 8 to each well. The absorbance was measured at $450 \mathrm{~nm}$ using EnSpire Multimode plate reader (PerkinElmer, Inc., Waltham, MA, USA). The optical density (OD) was calculated for cell viability assay. Cell viability $(\%)=\mathrm{OD}\left(_{\text {treated }}\right) / \mathrm{OD}\left(_{\text {control }}\right) \times 100 \%$.

Wound healing assay. Cell migration was measured using a wound healing assay. The MDA-MB-231 and MCF7 cells were cultured in 6-well plates. A clean pipette tip was used to inflict a 'wound' when cells formed a confluent monolayer, and the cultured cells in DMEM were supplemented with 2\% FBS. The cells were left untreated or treated with $\mathrm{HM}$ at 50, 100 or $150 \mu \mathrm{M}$. Images of the wound margins were captured using an inverted light microscope (Olympus Corporation, Tokyo, Japan) formed $0 \mathrm{~h}$ at this time point. Following incubation for $24 \mathrm{~h}$, images of the same region of cells were captured for measurement. The wound healing rate was calculated using the following formula: (average wound margin in $0 \mathrm{~h}$ - average wound margin in $24 \mathrm{~h}$ ) / average wound margin in $0 \mathrm{~h}$.

DAPI staining. The cells were left untreated or treated with $\mathrm{HM}$ at 50,100 or $150 \mu \mathrm{M}$ for $24 \mathrm{~h}$, washed twice with phosphate buffer saline (PBS), fixed in methanol for $10 \mathrm{~min}$ and finally stained with DAPI (cat. no. C1002; Beyotime Institute of Biotechnology, Haimen, China) for $10 \mathrm{~min}$ at room temperature. Images were then captured by fluorescent microscopy (Olympus Corporation).

Flow cytometry. Cell apoptosis was analyzed using a PE Annexin V apoptosis detection kit I (cat. no. 559763; BD Biosciences, USA). The cells were seeded in 6-well plates with 50, 100 and $150 \mu \mathrm{M}$ HM for $24 \mathrm{~h}$, and stained according 


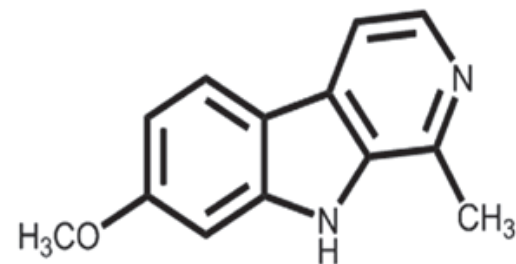

Figure 1. Structure of harmine.

to the manufacturer's instructions. The apoptotic cells were immediately detected by a FACS CaliberIISorter and the Cell Quest FACS system (BD Biosciences). Data were analyzed using FlowJo software (version 7.6.5; Tree Star, Inc., Ashland, OR, USA).

Western blotting. The cells were lysed in cell lysis buffer (cat. no. P0013B; Beyotime Institute of Biotechnology) and centrifuged $\left(12,000 \mathrm{x} \mathrm{g}, 10 \mathrm{~min}, 4^{\circ} \mathrm{C}\right)$ to collect the supernatants following treatment with $\mathrm{HM}$ (50, 100 or $150 \mu \mathrm{M}$ ) for $24 \mathrm{~h}$. The protein concentration was measured using a BCA Protein Assay kit (cat. no. CW0014S; CWBio, Beijing, China), and an equal quantity (20 $\mu \mathrm{g}$ per well) of protein was separated by $10 \%$ SDS-PAGE and transferred onto nitrocellulose membranes. The membranes were blocked using 5\% nonfat skim milk for $1 \mathrm{~h}$ at room temperature and then incubated with primary antibodies overnight for $4^{\circ} \mathrm{C}$. The primary antibodies included anti-TAZ (1:1,000; cat. no. 70148; Cell Signaling Technology, Inc., Danvers, MA, USA), anti-Bax (1:1,000; cat. no. ab-32503; Abcam, Cambridge, MA, USA), anti-Bcl-2 (1:1,000; cat. no. ab-32124; Abcam), anti-Erk (1:500; cat. no. sc-514302; Santa Cruz Biotechnology, Inc., Dallas, TX, USA), anti-phosphorylated (p-)Erk (1:500; cat. no. sc-81492; Santa Cruz Biotechnology, Inc.), anti-Akt (1:500; cat. no. sc-24500; Santa Cruz Biotechnology, Inc.), anti-p-Akt (1:500; cat. no. sc-7985; Santa Cruz Biotechnology, Inc.) and anti- $\beta$-actin (1:1,000; cat. no. sc-47778; Santa Cruz Biotechnology, Inc.). Following washing with TBST three times, for $10 \mathrm{~min}$ each time, goat anti-rabbit $\operatorname{IgG}(1: 10,000$; cat. no. sc-2040; Santa Cruz Biotechnology, Inc.) or goat anti-mouse IgG antibody (1:10,000; cat. no. sc-2005; Santa Cruz Biotechnology, Inc.) conjugated with horseradish peroxidase was incubated for $1 \mathrm{~h}$ at room temperature. The protein bands were visualized using the enhanced ECL Select Western Blotting Detection Reagent (cat. no. RPN2235; GE Healthcare Life Sciences, Chalfont, UK).

$R N A$ extraction and reverse transcription-quantitative polymerase chain reaction $(R T-q P C R)$ analysis. Total RNA was extracted from untreated and treated cells using TRIzol reagent (cat. no. 15596018; Thermo Fisher Scientific, Inc.) and cDNA was synthesized using the ReverTra Ace qPCR RT kit (cat. no. FSQ-101; Toyobo Life Science, Osaka, Japan). The qPCR was performed using UltraSYBR mixture (cat. no. CW-2601; CWBio) on an ABI StepOne Plus QPCR system (Thermo Fisher Scientific, Inc.). The thermocycling steps were as follows: Initial denaturation at $95^{\circ} \mathrm{C}$ for $10 \mathrm{~min}$, followed by 40 cycles at $95^{\circ} \mathrm{C}$ for $15 \mathrm{sec}, 58^{\circ} \mathrm{C}$ for $1 \mathrm{~min}$, and a final extension step at $72^{\circ} \mathrm{C}$ for $5 \mathrm{~min}$. The $2^{-\Delta \Delta \mathrm{Cq}}$ method was used to calculate changes in relative mRNA expression levels (35).
The primer sequences used were as follows: TAZ, forward 5'-GTCACCAACAGTAGCTCAGATC-3' and reverse 5'-AGT GATTACAGCCAGGTTAGAAAG-3'; $\beta$-actin, forward 5'-AC TCTTCCAGCCTTCCTTCC-3' and reverse 5'-CGTCATACT CCTGCTTGCTG-3'.

Xenograft mice model. A total of 20 six-week-old male athymic nude BALB/c mice (weight, 16-18 g) purchased from Beijing Vital River Laboratory Animal Technology (Beijing, China) were housed under specific pathogen-free conditions $\left(24-26^{\circ} \mathrm{C}\right.$; 12 -h light/dark cycle; free access to food and water). The mice were subcutaneously inoculated with MCF-7 cells $\left(1 \times 10^{7}\right)$ resuspended in $0.1 \mathrm{ml}$ DMEM. When the tumor size reached $100 \mathrm{~mm}^{3}$, the mice were randomly divided into four groups ( $n=5 /$ group) to receive one of the following treatments: Normal saline (NS; intraperitoneal injection) or 20,40 or $80 \mathrm{mg} / \mathrm{kg} /$ day of HM (5 days per week for 2 weeks, intraperitoneal injection). Tumor length (L) and width (W) were measured at 2-day intervals using a Vernier caliper, and tumor volume (V) was calculated using the following formula: $\mathrm{V}\left(\mathrm{mm}^{3}\right)=\mathrm{L}(\mathrm{mm}) \times \mathrm{W}^{2}\left(\mathrm{~mm}^{2}\right) \times 0.5$. After 4 weeks injection, all mice were sacrificed and tumor tissues were removed. Each mouse bore a single tumor, the maximum diameter of a single subcutaneous tumor reached $15.98 \mathrm{~mm}$, the maximum tumor volume reached $1,189 \mathrm{~mm}^{3}$. Tumor tissues from the mice were dissected for western blotting, RT-qPCR analysis and immunohistochemistry (IHC). The animal experiments were approved by the Committee on the Ethics of Animal Experiments of the Tongji Medical College, Huazhong University of Science and Technology (Wuhan, China; IACUC no. 837).

IHC analysis. IHC was performed to detect the levels of TAZ, Bcl-2, Bax, p-Erk and p-Akt in mouse tumor tissues. The samples were embedded in paraffin and sliced into thin sections (5 $\mu \mathrm{m})$. The sections were dewaxed in xylene and sequentially rehydrated with 100, 95, 80 and $75 \%$ ethanol. The slides were blocked with BSA for $2 \mathrm{~h}$ at room temperature. The slides were incubated with primary antibodies anti-TAZ, Bcl-2, Bax, p-Erk and p-Akt (1:200) overnight at $4^{\circ} \mathrm{C}$. Following washing three times with PBS for $10 \mathrm{~min}$ each time, biotinylated goat anti-rabbit secondary antibody (1:200; cat. no. A0279; Beyotime Institute of Biotechnology) was incubated for $1 \mathrm{~h}$ at $37^{\circ} \mathrm{C}$, and diaminobenzidine tetrachloride (cat. no. P0203; Beyotime Institute of Biotechnology) was used for staining at $37^{\circ} \mathrm{C}$ for $10 \mathrm{~min}$. Data were analyzed using a light microscope (Olympus Corporation).

Statistical analysis. Statistical analysis was performed by Student's t-test for comparison between two groups or one-way ANOVA for comparison between more than two groups. The least-significant difference post hoc test was used following ANOVA. All statistical analyses were performed using SPSS 17.0 software (SPSS, Inc., Chicago, IL, USA). P<0.05 was considered to indicate a statistically significant difference.

\section{Results}

$H M$ inhibits proliferation and migration, and induces apoptosis in MDA-MB-231 and MCF-7 cells. The 
A

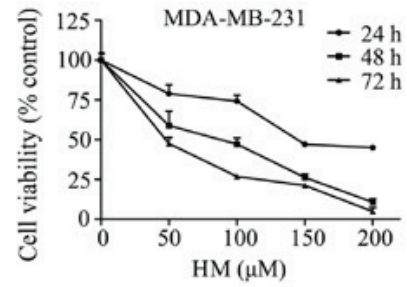

B

$\mathrm{C}$
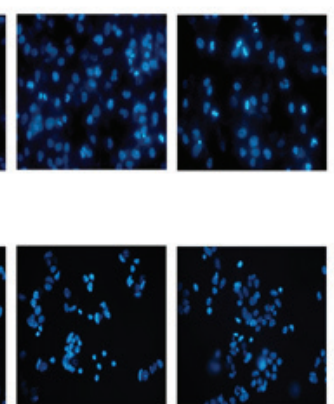

100

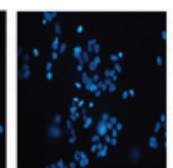

150
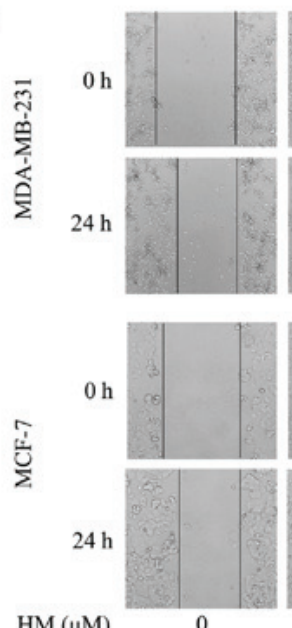

$\mathrm{HM}(\mu \mathrm{M})$
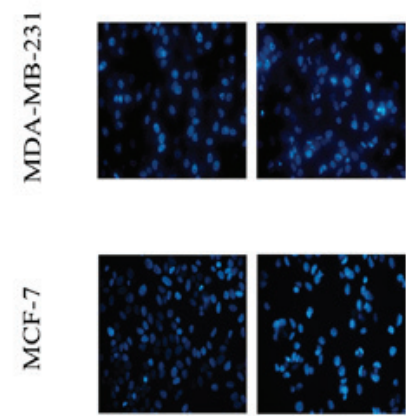

$\mathrm{HM}(\mu \mathrm{M})$
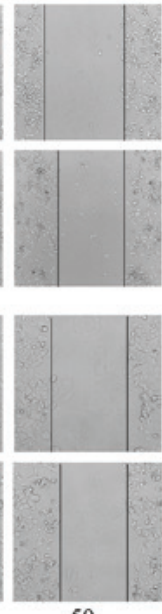

50

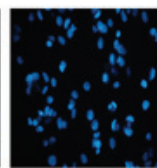

50

$\mathrm{D}$

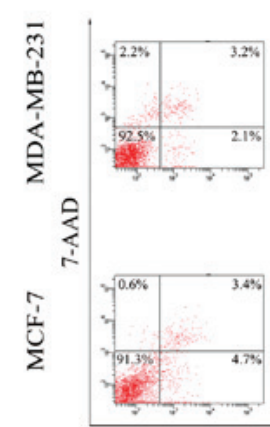

$\mathrm{HM}(\mu \mathrm{M})$
0

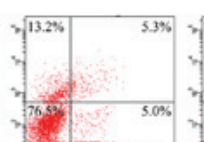

50
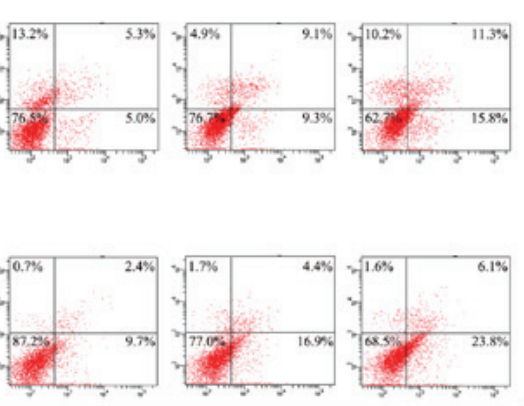

Annexin V-PE

100

150
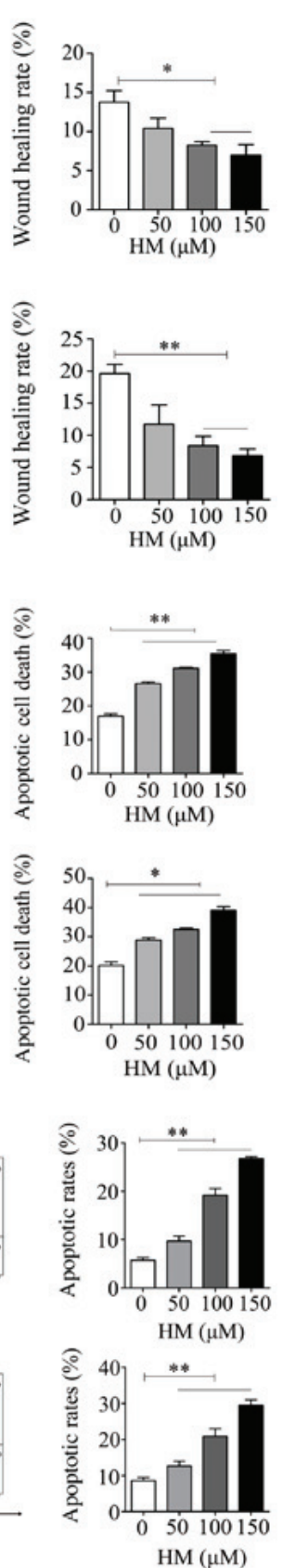

Figure 2. HM inhibits proliferation and migration, and induces apoptosis of MDA-MB-231 and MCF-7 cells. (A) Dose- and time-dependent effects of HM on MDA-MB-231 and MCF-7 cell viability. Cells were treated with 50, 100 or $150 \mu \mathrm{M}$ HM for 24, 48 and 72 h, and cell viability was measured via CCK-8 assay. Data are presented as the mean \pm SD. (B) MDA-MB-231 and MCF-7 cells were treated with HM (50, 100 or $150 \mu$ M) for 24 h. Cell migration was detected using a monolayer wound healing assay (magnification, $\mathrm{x} 400$ ). The wound healing rate at $24 \mathrm{~h}$ was quantified. (C) Apoptotic morphology of the MDA-MB-231 and MCF-7 cells was detected by fluorescent microscopy following DAPI staining (magnification, x400). The histogram represents the apoptotic rate. (D) Analysis of apoptotic cells induced by HM for $24 \mathrm{~h}$ using flow cytometry. The histogram represents the apoptotic rates. Data are presented as the mean \pm SD from three independent experiments. ${ }^{*} \mathrm{P}<0.05$ and ${ }^{* *} \mathrm{P}<0.01$, with comparisons indicated by brackets. HM, harmine.

anti-proliferative effect of HM on breast cancer cells was evaluated via CCK-8 assay. The MDA-MB-231 and MCF-7 cells were treated with different concentrations of HM for 24 ,
48 and 72 h, respectively. As shown in Fig. 2A, HM inhibited cell proliferation in a dose- and time-dependent manner. In addition to inhibiting cell proliferation, HM suppressed 
A

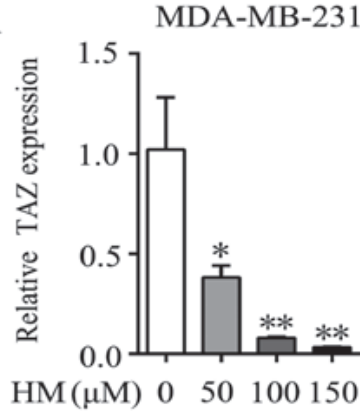

B

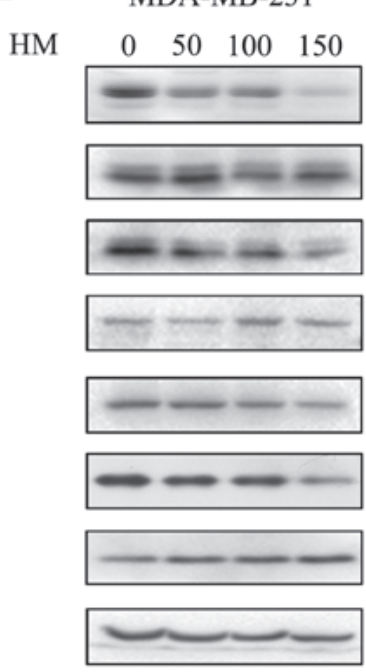

MCF-7

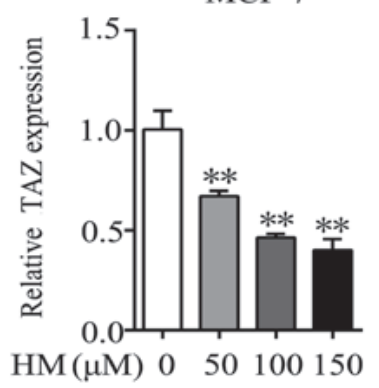

MCF-7

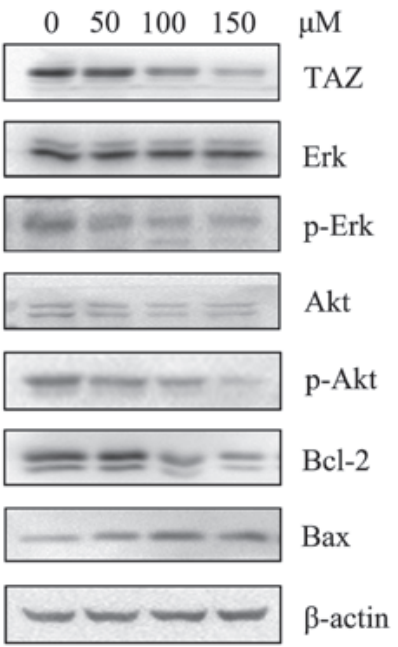

Figure 3. HM mediates antitumor effects via the downregulation of TAZ in MDA-MB-231 and MCF-7 cells. (A) MDA-MB-231 and MCF-7 cells were treated with $\mathrm{HM}(50,100$ or $150 \mu \mathrm{M})$ for $24 \mathrm{~h}$ and the relative mRNA transcription levels were determined via reverse transcription-quantitative polymerase chain reaction analysis. (B) MDA-MB-231 and MCF-7 cells were treated with $\mathrm{HM}(50,100$ or $150 \mu \mathrm{M})$ for $48 \mathrm{~h}$, and the expression of proliferation, migration and apoptosis-related proteins, Akt, p-Akt, Erk, p-Erk, Bcl-2 and Bax, were detected by western blotting. $\beta$-actin was used as an internal standard. Data are presented as the mean $\pm \mathrm{SD}$ of three independent experiments. "P<0.05 and ${ }^{* * *} \mathrm{P}<0.01$ vs. $0 \mu \mathrm{M}$. HM, harmine; TAZ, transcriptional co-activator with PDZ-binding motif; Akt, protein kinase B; Erk, extracellular signal-regulated kinase; Bcl-2, B-cell lymphoma 2; Bax, Bcl-2-associated X protein; p-, phosphorylated.

the migration of breast cancer cells. The MDA-MB-231 and MCF-7 cells were exposed to different concentrations of HM for $24 \mathrm{~h}$, as shown in Fig. 2B, and the results revealed a dose-dependent inhibition of cell migration. Apoptotic morphological changes were determined by DAPI staining. Chromatin condensation and nuclear fragmentation, which are characteristics of apoptosis, were evident in the MDA-MB-231 and MCF-7 cells treated with HM (50, 100 or $150 \mu \mathrm{M}$ ) for $24 \mathrm{~h}$. As shown in Fig. 2C, a significant increase of chromatin condensation and nuclear fragmentation was observed in the HM-treated cells, when compared with that of control (no HM added) cells. To further confirm the pro-apoptotic effect of HM, cell apoptosis was determined by flow cytometry using a Annexin $\mathrm{V}$ apoptosis detection kit. Following treatment of the MDA-MB-231 cells with $\mathrm{HM}$ at 50,100 or $150 \mu \mathrm{M}$ for $24 \mathrm{~h}$, the apoptotic population increased from 5.7 to $26.7 \%$ in a dose-dependent manner. Similar results were observed in MCF-7 cells (Fig. 2D). In combination, these results indicated that HM had an anticancer effect on the breast cancer cell lines.

$H M$ decreases the expression level of TAZ in MDA-MB-231 and $M C F-7$ cells. Increasing studies have suggested that TAZ is an oncogene in human cancer cells and serves an important role in the development of tumors (17). It has been reported that TAZ is expressed at high levels in breast cancer cell lines, promotes cell proliferation, migration and tumorigenesis, and inhibits apoptosis in breast cancer $(29,30,34)$. To further understand the mechanism underlying the anticancer activities induced by HM, the present study examined whether TAZ was involved in the anticancer mechanism. The relative mRNA expression of TAZ was significantly decreased, compared with that in the control group (Fig. 3A). Following $48 \mathrm{~h}$ of HM treatment (50, 100 or $150 \mu \mathrm{M}$ ), the protein expression of TAZ was reduced in the MDA-MB-231 and MCF-7 cells. The activation of MAPK kinase/ERK and PI3K/AKT has been reported to regulate cancer cell proliferation and migration through various pathways $(36,37)$. In the present study, proliferation- and migration-related proteins, including $\mathrm{p}$-Erk and $\mathrm{p}$-Akt, were decreased, whereas anti-apoptotic protein $\mathrm{Bcl}-2$ was decreased and pro-apoptotic Bax was increased (Fig. 3B).

Overexpression of TAZ inhibits the anticancer activity of HM. In order to validate that HM-induced anticancer activity via targeting TAZ, the effect of increased TAZ on HM-mediated anticancer activity was measured. The MDA-MB-231 and MCF-7 cells were transfected with either a TAZ-expressing plasmid (pCMV6-TAZ) or a control plasmid (pCMV6-control). 
A
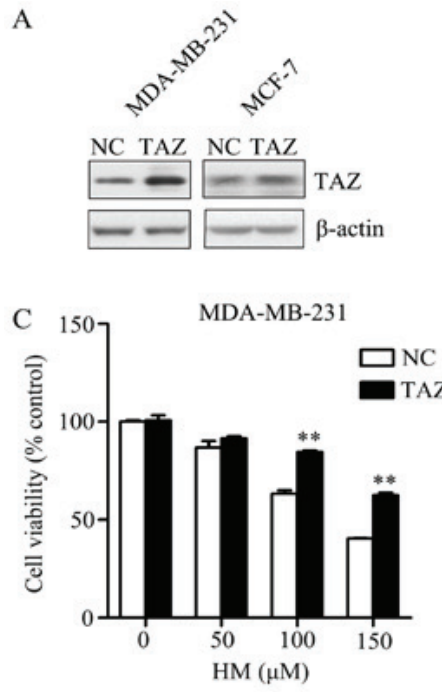

D $\quad{ }_{\mathrm{NC}}^{\mathrm{HM}-50 \mu \mathrm{M}}$
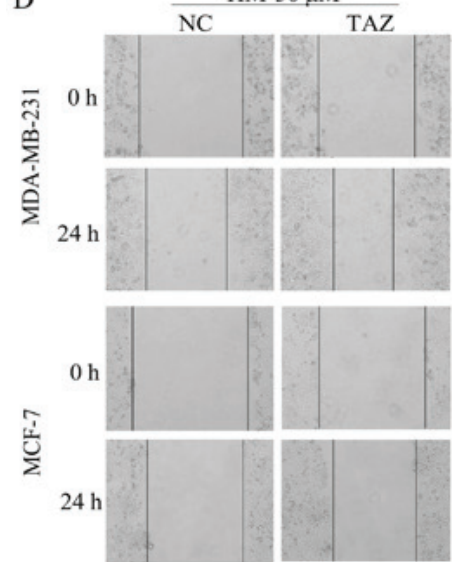

$\mathrm{E}$

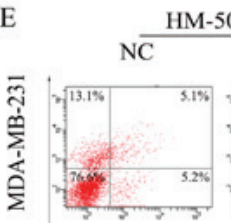

$\frac{\mathrm{HM}-50 \mu \mathrm{M}}{\mathrm{CAZ}}$

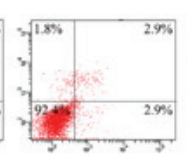

定

i

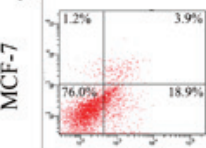

B
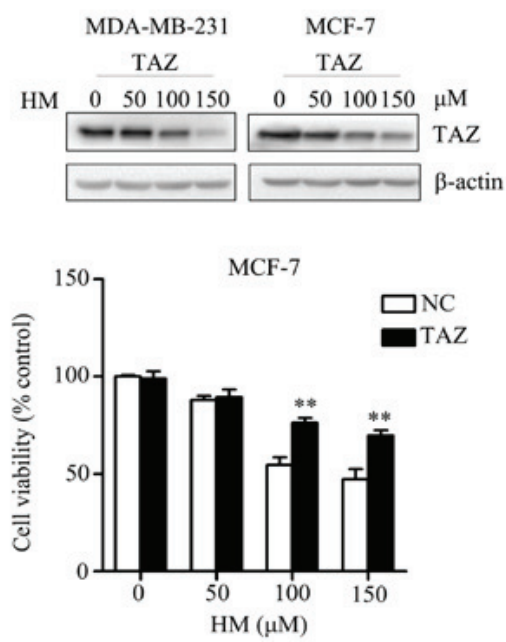
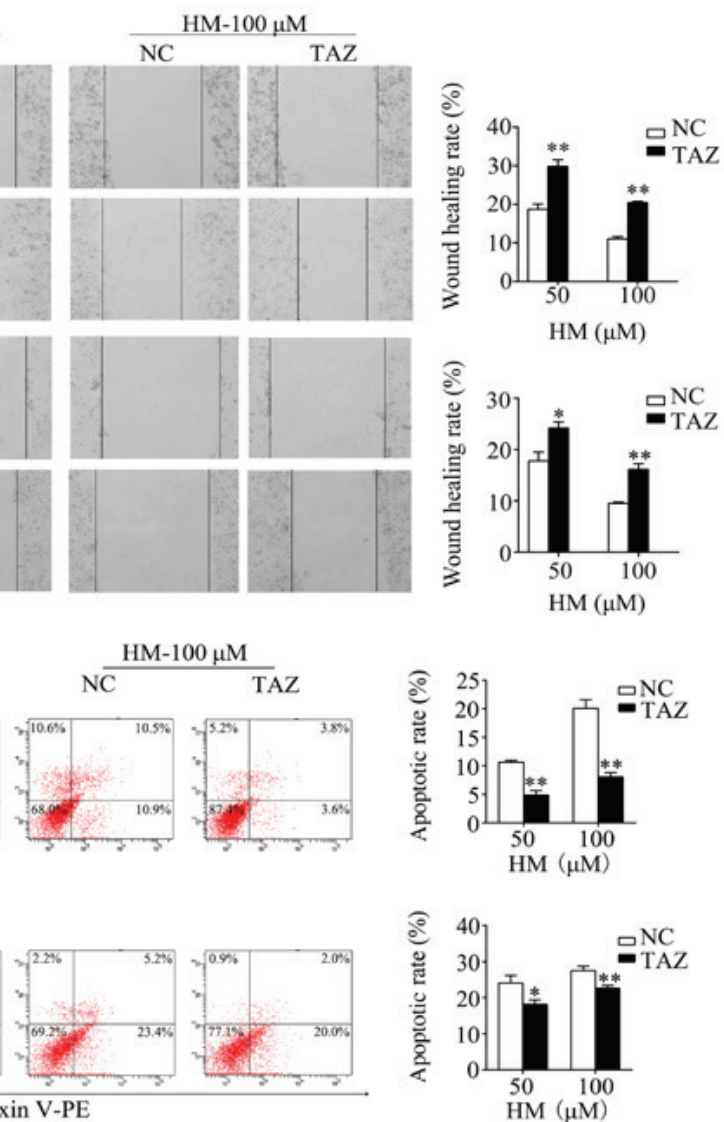

Figure 4. Overexpression of TAZ inhibits the anticancer activities of HM. (A) Transfection efficiency of TAZ in MDA-MB-231 and MCF-7 cells was determined by western blotting. (B) Western blotting results of the TAZ-overexpression groups under HM treatment (50, 100 and $150 \mu \mathrm{M}$ ). (C) Overexpression of TAZ inhibited the HM-induced inhibition of proliferation. (D) Overexpression of TAZ inhibited the HM-induced inhibition of migration activity. (E) Overexpression of TAZ inhibited HM-induced apoptosis. Data are presented as the mean $\pm \mathrm{SD}$ of three independent experiments. ${ }^{*} \mathrm{P}<0.05$ and ${ }^{* *} \mathrm{P}<0.01$ vs. NC. TAZ, transcriptional co-activator with PDZ-binding motif; HM, harmine; NC, negative control (pCMV6-control).

At 2 days post-transfection, western blotting was performed to check the transfection efficiency of TAZ, the expression of TAZ was increased in the TAZ overexpression group (Fig. 4A). The expression level of TAZ in the TAZ overexpression group under HM treatment $(50,100$ or $150 \mu \mathrm{M})$ was decreased in a dose-dependent manner (Fig. 4B). The transfected cells were treated with HM $(50,100$ or $150 \mu \mathrm{M})$ for $48 \mathrm{~h}$. When compared with negative control cells, the overexpression of TAZ in breast cancer cells was found to prevent the HM-induced inhibition of cell proliferation and migration (Fig. 4C and D). HM-induced cell apoptosis was also inhibited by the overexpression of TAZ (Fig. 4E).

HM inhibits the growth of breast xenograft tumors in vivo. The MCF-7 cells were subcutaneously injected into athymic nude mice to establish a breast xenograft tumor model, and tumor growth was recorded. Different doses of HM (20, 40 or $80 \mathrm{mg} / \mathrm{kg} /$ day) were administered, and HM was shown 
A

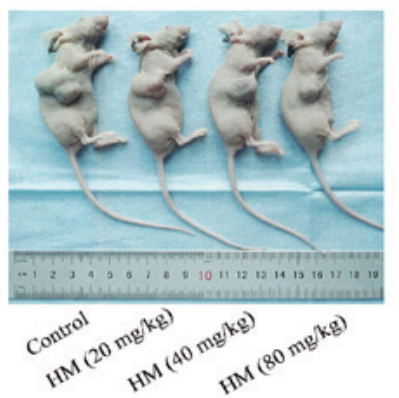

C

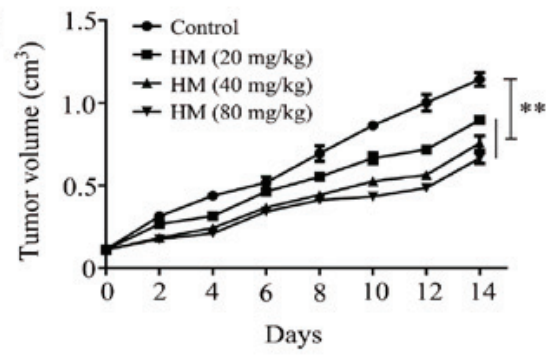

$\mathbf{E}$

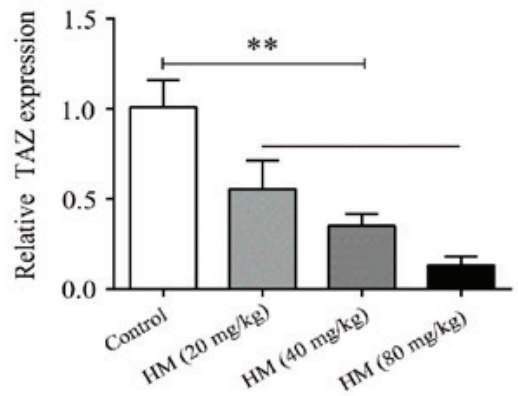

G

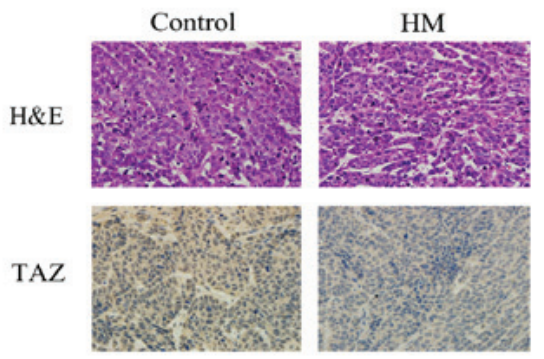

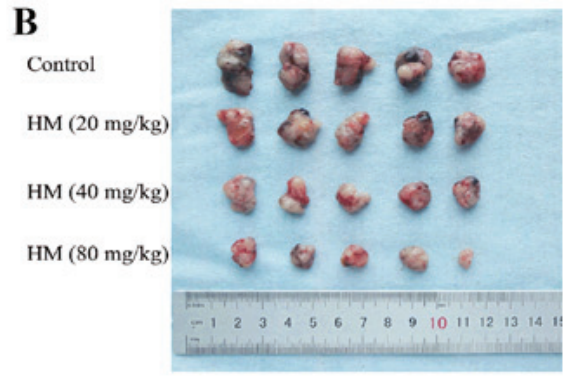

D

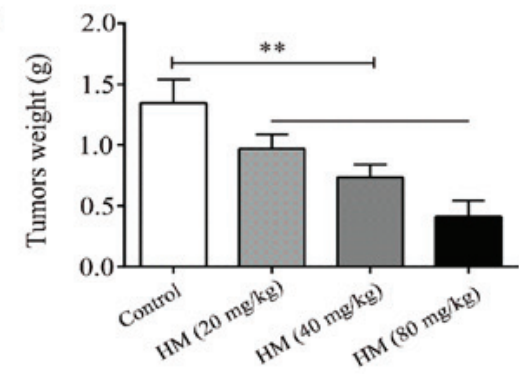

$\mathbf{F}$

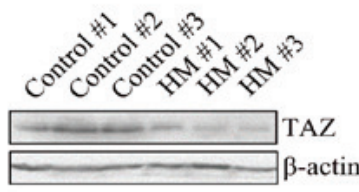

H
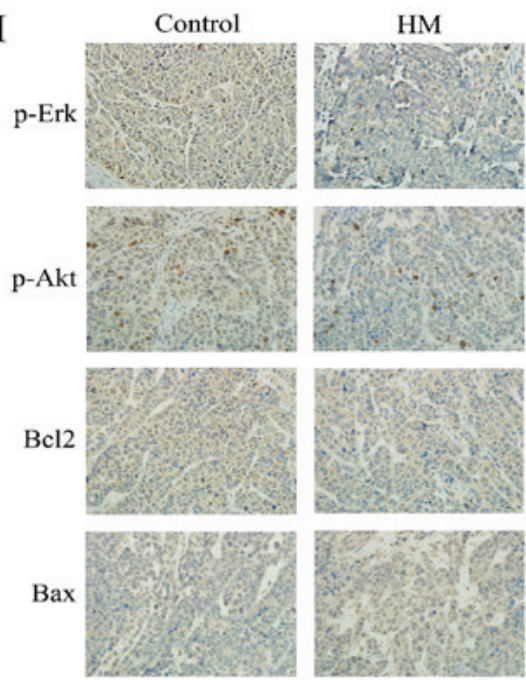

Figure 5. HM inhibits breast cancer tumor growth in vivo. (A) Mice were sacrificed 2 weeks after being injected with different doses of HM. (B) Tumors were dissected and images were captured. (C) MCF-7 cells were injected into the nude mice. Tumor volume was measured at 2-day intervals and the growth curves were drafted. (D) Tumors were weighted at the end of treatment. (E) Relative mRNA expression of TAZ in tumor tissues from xenograft mice. (F) Representative images from western blot analysis for TAZ protein expression in tumor tissues from xenograft mice. (G and $\mathrm{H}$ ) Tumor tissues were analyzed by $\mathrm{H} \& \mathrm{E}$ and immunohistochemistry for TAZ, Bax, Bcl-2, p-Erk and p-Akt. Magnification, $\mathrm{x} 400$. ${ }^{* *} \mathrm{P}<0.01$, with comparisons indicated by brackets. HM, harmine; H\&E, hematoxylin and eosin; TAZ, transcriptional co-activator with PDZ-binding motif; Akt, protein kinase B; Erk, extracellular signal-regulated kinase; Bcl-2, B-cell lymphoma 2; Bax, Bcl-2-associatedX protein; p-, phosphorylated.

to reduce tumor volume and weight in a dose- and timedependent manner (Fig. 5A-D). Tumor tissues were used to evaluate the level of TAZ by IHC, RT-qPCR and western blot analyses. It was found that HM decreased the level of TAZ, compared with that in the control group (Fig. 5E-G). The
IHC results showed that the proliferation and expression of metastasis-related proteins, including $\mathrm{p}$-Akt and p-Erk, were decreased compared with the control group (Fig. 5H). These results indicated that HM inhibited the development of breast cancer in vivo. 


\section{Discussion}

Although current therapeutic strategies can lead to considerable anticancer activity, the development of novel antitumor agents with less toxicity remains of interest. HM is a natural $\beta$-carboline alkaloid isolated from Peganum harmala, which was been previously used in folk medicine as an anticancer therapy (5). Studies have shown the anticancer activities of HM through the activation of apoptosis and autophagy (38); however, the effect of HM targeting TAZ in breast cancer has not been previously reported. The present study demonstrated the anticancer effects of HM on breast cancer and revealed a novel target of HM that is important for its activity.

Previous studies have reported the antitumor effects of HM on hepatocellular carcinoma (39), gastric cancer (9) and neuroblastoma (40). In the present study, the anticancer activity of HM was demonstrated using MDA-MB-231 and MCF-7 cells in vitro and MCF-7 cell-derived xenograft tumor in vivo. In vitro, $\mathrm{HM}$ was found to inhibit proliferation and migration in a dose- and time- dependent manner. Consistent with this result, it was observed that HM significantly increased cell apoptosis. Following treatment with HM, cellular morphological changes, including cell rounding, shrinkage and karyopyknosis, were observed. In vivo, HM also inhibited tumor growth, in terms of tumor volume and weight, in a dose-dependent manner. These results suggest that HM may be used in combination with other chemical drugs for the inhibition of breast cancer growth and metastasis.

To reveal the underlying molecular mechanism through which $\mathrm{HM}$ induces anticancer activity in human breast cancer, the expression of TAZ was investigated following HM treatment. The Hippo pathway serves an important role in mammalian developmental stages (41-44). TAO1-3, mammalian Ste20-like kinase 1/2, MAPK kinase kinase kinase $1-4 / 6 / 7$, large tumor suppressor $1 / 2$ and nuclear Dbf2-related 1/2 are the core kinases, whereas yes-associated protein (YAP) and TAZ are the primary downstream effectors of the Hippo pathway in mammals. When phosphorylated by these core kinases, YAP is sequestered and degraded in the cytoplasm (45). The Hippo pathway coordinates cell proliferation and apoptosis in response to a variety of signals by regulating the transcriptional coactivators YAP and TAZ (46). Although it has been reported that YAP functions as a tumor suppressor in breast cancer (47), it has been described that TAZ is an oncogenic protein in certain types of human cancer, including non-small cell lung and breast cancer $(26,34)$. It was also found in the present study that the level of TAZ was decreased in vitro following treatment with $\mathrm{HM}$, and that the expression of TAZ was reduced in the MCF-7 xenograft tumor. These results revealed that HM may function by targeting TAZ. To further verify this, experiments were performed to reveal that the antiproliferative and pro-apoptotic activities of HM were inhibited by overexpressing TAZ.

Furthermore, several growth factors associated with proliferation and metabolism were examined in HM-treated MDA-MB-231 and MCF-7 cells. The PI3K/Akt pathway is commonly recognized for its critical role in autophagy (48), metabolism (49) and metastasis (50). The elevated phosphorylation of Akt led to higher tumor recurrence and poorer overall survival (51). Erk belongs to a subclass of MAPKs that includes serine/threonine kinases. Upon activation of the MAPK pathway, Erk is phosphorylated and regulates various cellular processes, including proliferation, differentiation, apoptosis and transformation (52). In the present study, it was found that HM treatment increased the levels of p-Akt and p-Erk in vitro and in vivo. The Bcl-2 family of proteins is divided into two subfamilies; Bcl-2 and Bcl-xl are anti-apoptotic proteins, whereas Bax, Bad and Bid are pro-apoptotic proteins (53). The results of the present study indicated a decreased level of Bcl-2 and increased level of Bax in HM-treated cells, which is consistent with the immunohistochemical results obtained from the MCF-7 xenograft tumor. All the above results confirmed the antiproliferative and pro-apoptotic function of $\mathrm{HM}$ on breast cancer.

In conclusion, the present study demonstrated the anticancer (antiproliferative, antimetastatic and pro-apoptotic) activity of HM on breast cancer cell lines. The study also identified a novel target of HM, TAZ, in antagonizing the role of HM on breast cancer cell lines, however, the anticancer molecular mechanisms of HM require further in-depth investigation. Therefore, HM may serve as an anticancer agent in the chemoprevention and treatment of breast cancer, although future clinical and pharmacological studies are required to confirm this.

\section{Acknowledgements}

The authors would like to thank Mrs. Weiqun Chen (Central Laboratory, The Central Hospital of Wuhan) for kindly providing technical assistance in cell experiments and $\mathrm{Mr}$. Shunchang Zhou (Department of Experimental Zoology, Tongji Medical College) for animal care.

\section{Funding}

This study was partially sponsored by the National Natural Science Foundation of China (grant no. 81301084 to CP; grant no. 81800296 to SC), the Natural Science Foundation of Hubei Province in China (grant no. 2017CFB357 to SC) and the Health Commission Foundation of Hubei Province in China (grant no. WJ2019M029 to JHu).

\section{Availability of data and materials}

The datasets used and analyzed during the current study are available from the corresponding author on reasonable request.

\section{Authors' contributions}

$\mathrm{SC}, \mathrm{CP}, \mathrm{YD}, \mathrm{JHe}$ and JHu conceived and designed the experiments. YD, JHe, TZ and GY performed the experiments. XS and TY analyzed data. YD and JHe wrote the manuscript.

\section{Ethics approval and consent to participate}

Animal studies were approved by the Committee on the Ethics of Animal Experiments of the Tongji Medical College, Huazhong University of Science and Technology (IACUC no. 837). 


\section{Patient consent for publication}

Not applicable.

\section{Competing interests}

The authors declare that they have no competing interests.

\section{References}

1. Siegel RL, Miller KD and Jemal A: Cancer statistics, 2016. CA Cancer J Clin 66: 7-30, 2016.

2. Chang L, Weiner LS, Hartman SJ, Horvath S, Jeste D, Mischel PS and Kado DM: Breast cancer treatment and its effects on aging. J Geriatr Oncol 10: 346-355, 2019.

3. Nathanson KL and Domchek SM: Therapeutic approaches for women predisposed to breast cancer. Annu Rev Med 62: 295-306, 2011.

4. Patel K, Gadewar M, Tripathi R, Prasad SK and Patel DK: A review on medicinal importance, pharmacological activity and bioanalytical aspects of beta-carboline alkaloid 'Harmine'. Asian Pac J Trop Biomed 2: 660-664, 2012.

5. Berrougui H, Martín-Cordero C, Khalil A, Hmamouchi M, Ettaib A, Marhuenda E and Herrera MD: Vasorelaxant effects of harmine and harmaline extracted from Peganum harmala $\mathrm{L}$. seeds in isolated rat aorta. Pharmacol Res 54: 150-157, 2006.

6. Chen Q, Chao R, Chen H, Hou X, Yan H, Zhou S, Peng W and $\mathrm{Xu} \mathrm{A}$ : Antitumor and neurotoxic effects of novel harmine derivatives and structure-activity relationship analysis. Int $\mathrm{J}$ Cancer 114: 675-682, 2005.

7. Zhang H, Sun K, Ding J, Xu H, Zhu L, Zhang K, Li X and Sun W: Harmine induces apoptosis and inhibits tumor cell proliferation, migration and invasion through down-regulation of cyclooxygenase-2 expression in gastric cancer. Phytomedicine 21: 348-355, 2014.

8. Dai F, Chen Y, Song Y, Huang L, Zhai D, Dong Y, Lai L, Zhang T, Li D, Pang X, et al: A natural small molecule harmine inhibits angiogenesis and suppresses tumour growth through activation of p53 in endothelial cells. PLoS One 7: e52162, 2012.

9. Li C, Wang Y, Wang C, Yi X, Li M and He X: Anticancer activities of harmine by inducing a pro-death autophagy and apoptosis in human gastric cancer cells. Phytomedicine 28: 10-18, 2017.

10. Abe A and Yamada H: Harmol induces apoptosis by caspase- 8 activation independently of Fas/Fas ligand interaction in human lung carcinoma H596 cells. Anticancer Drugs 20: 373-381, 2009.

11. Hashemi Sheikh Shabani S, Seyed Hasan Tehrani S, Rabiei Z, Tahmasebi Enferadi S and Vannozzi GP: Peganum harmala L.'s anti-growth effect on a breast cancer cell line. Biotechnol Rep (Amst) 8: 138-143, 2015.

12. Zhang L, Zhang F, Zhang W, Chen L, Gao N, Men Y, Xu X and Jiang Y: Harmine suppresses homologous recombination repair and inhibits proliferation of hepatoma cells. Cancer Biol Ther 16 $1585-1592,2015$

13. Hamsa TP and Kuttan G: Harmine activates intrinsic and extrinsic pathways of apoptosis in B16F-10 melanoma. Chin Med 6: 11, 2011.

14. Song Y, Kesuma D, Wang J, Deng Y, Duan J, Wang JH and Qi RZ: Specific inhibition of cyclin-dependent kinases and cell proliferation by harmine. Biochem Biophys Res Commun 317: 128-132, 2004.

15. Zou N, Wei Y, Li F, Yang Y, Cheng X and Wang C: The inhibitory effects of compound Muniziqi granule against B16 cells and harmine induced autophagy and apoptosis by inhibiting Akt/mTOR pathway. BMC Complement Altern Med 17: 517, 2017.

16. Hamsa TP and Kuttan G: Harmine inhibits tumour specific neo-vessel formation by regulating VEGF, MMP, TIMP and pro-inflammatory mediators both in vivo and in vitro. Eur J Pharmacol 649: 64-73, 2010.

17. Zhou X and Lei QY: Regulation of TAZ in cancer. Protein Cell 7: 548-561, 2016.

18. Park KS, Whitsett JA, Di Palma T, Hong JH, Yaffe MB and Zannini M: TAZ interacts with TTF-1 and regulates expression of surfactant protein-C. J Biol Chem 279: 17384-17390, 2004.

19. Jeong H, Bae S, An SY, Byun MR, Hwang JH, Yaffe MB, Hong JH and Hwang ES: TAZ as a novel enhancer of MyoD-mediated myogenic differentiation. FASEB J 24: 3310-3320, 2010.
20. Varelas X, Samavarchi-Tehrani P, Narimatsu M, Weiss A Cockburn K, Larsen BG, Rossant J and Wrana JL: The Crumbs complex couples cell density sensing to Hippo-dependent control of the TGF- $\beta$-SMAD pathway. Dev Cell 19: 831-844, 2010

21. Cui CB, Cooper LF, Yang X, Karsenty G and Aukhil I: Transcriptional coactivation of bone-specific transcription factor Cbfa1 by TAZ. Mol Cell Biol 23: 1004-1013, 2003.

22. Mahoney WM Jr, Hong JH, Yaffe MB and Farrance IK: The transcriptional co-activator TAZ interacts differentially with transcriptional enhancer factor-1 (TEF-1) family members. Biochem J 388: 217-225, 2005.

23. Hong JH, Hwang ES, McManus MT, Amsterdam A, Tian Y, Kalmukova R, Mueller E, Benjamin T, Spiegelman BM, Sharp PA, et al: TAZ, a transcriptional modulator of mesenchymal stem cell differentiation. Science 309: 1074-1078, 2005.

24. Zhang H, Liu CY, Zha ZY, Zhao B, Yao J, Zhao S, Xiong Y, Lei QY and Guan KL: TEAD transcription factors mediate the function of TAZ in cell growth and epithelial-mesenchymal transition. J Biol Chem 284: 13355-13362, 2009.

25. Hong JH and Yaffe MB: TAZ: A beta-catenin-like molecule that regulates mesenchymal stem cell differentiation. Cell Cycle 5: 176-179, 2006.

26. Zhou Z, Hao Y, Liu N, Raptis L, Tsao MS and Yang X: TAZ is a novel oncogene in non-small cell lung cancer. Oncogene 30: 2181-2186, 2011.

27. Wang L, Shi S, Guo Z, Zhang X, Han S, Yang A, Wen W and Zhu Q: Overexpression of YAP and TAZ is an independent predictor of prognosis in colorectal cancer and related to the proliferation and metastasis of colon cancer cells. PLoS One 8: e65539, 2013.

28. Bhat KP, Salazar KL, Balasubramaniyan V, Wani K, Heathcock L, Hollingsworth F, James JD, Gumin J, Diefes KL, Kim SH, et al: The transcriptional coactivator TAZ regulates mesenchymal differentiation in malignant glioma. Genes Dev 25: 2594-2609, 2011.

29. Zhou X, Wang S, Wang Z, Feng X, Liu P, Lv XB, Li F, Yu FX, Sun Y, Yuan H, et al: Estrogen regulates Hippo signaling via GPER in breast cancer. J Clin Invest 125: 2123-2135, 2015.

30. Cordenonsi M, Zanconato F, Azzolin L, Forcato M, Rosato A, Frasson C, Inui M, Montagner M, Parenti AR, Poletti A, et al: The Hippo transducer TAZ confers cancer stem cell-related traits on breast cancer cells. Cell 147: 759-772, 2011.

31. Yang N, Morrison CD, Liu P, Miecznikowski J, Bshara W, Han S, Zhu Q, Omilian AR, Li X and Zhang J: TAZ induces growth factor-independent proliferation through activation of EGFR ligand amphiregulin. Cell Cycle 11: 2922-2930, 2012.

32. Xu W, Wei Y, Wu S, Wang Y, Wang Z, Sun Y, Cheng SY and Wu J: Up-regulation of the Hippo pathway effector TAZ renders lung adenocarcinoma cells harboring EGFR-T790M mutation resistant to gefitinib. Cell Biosci 5: 7, 2015.

33. Yuen HF, McCrudden CM, Huang YH, Tham JM, Zhang X, Zeng Q, Zhang SD and Hong W: TAZ expression as a prognostic indicator in colorectal cancer. PLoS One 8: e54211, 2013.

34. Chan SW, Lim CJ, Guo K, Ng CP, Lee I, Hunziker W, Zeng Q and Hong W: A role for TAZ in migration, invasion, and tumorigenesis of breast cancer cells. Cancer Res 68: 2592-2598, 2008.

35. Livak KJ and Schmittgen TD: Analysis of relative gene expression data using real-time quantitative PCR and the 2(-Delta Delta C(T)) Method. Methods 25: 402-408, 2001.

36. Chen H, Zhu G, Li Y, Padia RN, Dong Z, Pan ZK, Liu K and Huang S: Extracellular signal-regulated kinase signaling pathway regulates breast cancer cell migration by maintaining slug expression. Cancer Res 69: 9228-9235, 2009.

37. Pal I and Mandal M: PI3K and Akt as molecular targets for cancer therapy: Current clinical outcomes. Acta Pharmacol Sin 33: 1441-1458, 2012.

38. Geng X, Ren Y, Wang F, Tian D, Yao X, Zhang Y and Tang J: Harmines inhibit cancer cell growth through coordinated activation of apoptosis and inhibition of autophagy. Biochem Biophys Res Commun 498: 99-104, 2018.

39. Cao MR, Li Q, Liu ZL, Liu HH, Wang W, Liao XL, Pan YL and Jiang JW: Harmine induces apoptosis in HepG2 cells via mitochondrial signaling pathway. Hepatobiliary Pancreat Dis Int 10: 599-604, 2011.

40. Uhl KL, Schultz CR, Geerts D and Bachmann AS: Harmine, a dual-specificity tyrosine phosphorylation-regulated kinase (DYRK) inhibitor induces caspase-mediated apoptosis in neuroblastoma. Cancer Cell Int 18: 82, 2018.

41. Sasaki H: Roles and regulations of Hippo signaling during preimplantation mouse development. Dev Growth Differ 59: 12-20, 2017. 
42. He J, Bao Q, Yan M, Liang J,Zhu Y, Wang C and Ai D: The role of Hippo/yes-associated protein signalling in vascular remodelling associated with cardiovascular disease. $\mathrm{Br} \mathrm{J}$ Pharmacol 175: 1354-1361, 2018.

43. Patel SH, Camargo FD and Yimlamai D: Hippo Signaling in the Liver Regulates Organ Size, Cell Fate, and Carcinogenesis. Gastroenterology 152: 533-545, 2017.

44. Gregorieff A and Wrana JL: Hippo signalling in intestinal regeneration and cancer. Curr Opin Cell Biol 48: 17-25, 2017.

45. Watt KI, Harvey KF and Gregorevic P: Regulation of Tissue Growth by the Mammalian Hippo Signaling Pathway. Front Physiol 8: 942, 2017.

46. Kim W and Jho EH: The history and regulatory mechanism of the Hippo pathway. BMB Rep 51: 106-118, 2018.

47. Yuan M, Tomlinson V, Lara R, Holliday D, Chelala C, Harada T, Gangeswaran R, Manson-Bishop C, Smith P, Danovi SA, et al: Yes-associated protein (YAP) functions as a tumor suppressor in breast. Cell Death Differ 15: 1752-1759, 2008.

48. Yu X, Long YC and Shen HM: Differential regulatory functions of three classes of phosphatidylinositol and phosphoinositide 3-kinases in autophagy. Autophagy 11: 1711-1728, 2015.

49. Tang D, Chen QB, Xin XL and Aisa HA: Anti-diabetic effect of three new norditerpenoid alkaloids in vitro and potential mechanism via PI3K/Akt signaling pathway. Biomed Pharmacother 87: 145-152, 2017.
50. Lynch JT, McEwen R, Crafter C, McDermott U, Garnett MJ, Barry ST and Davies BR: Identification of differential PI3K pathway target dependencies in T-cell acute lymphoblastic leukemia through a large cancer cell panel screen. Oncotarget 7: 22128-22139, 2016

51. Li YH, Fu HL, Tian ML, Wang YQ, Chen W, Cai LL, Zhou XH and Yuan HB: Neuron-derived FGF10 ameliorates cerebral ischemia injury via inh+ibiting NF- $\kappa \mathrm{B}$-dependent neuroinflammation and activating PI3K/Akt survival signaling pathway in mice. Sci Rep 6: 19869, 2016

52. Fujimori Y, Inokuchi M, Takagi Y, Kato K, Kojima K and Sugihara K: Prognostic value of RKIP and p-ERK in gastric cancer. J Exp Clin Cancer Res 31: 30, 2012

53. Roos DH, Puntel RL, Lugokenski TH, Ineu RP, Bohrer D, Burger ME, Franco JL, Farina M, Aschner M, Rocha JB, et al: Complex methylmercury-cysteine alters mercury accumulation in different tissues of mice. Basic Clin Pharmacol Toxicol 107: 789-792, 2010.

(i) $($ This work is licensed under a Creative Commons Attribution-NonCommercial-NoDerivatives 4.0 International (CC BY-NC-ND 4.0) License. 